\section{pH triggered self-assembly of core cross-linked star polymers possessing thermoresponsive cores $\dagger$}

\author{
Alexander W. Jackson and David A. Fulton* \\ Received 29th March 2011, Accepted 21st April 2011 \\ DOI: $10.1039 / \mathrm{c} 1 \mathrm{cc} 11785 \mathrm{~h}$
}

Core cross-linked star polymers possessing responsiveness to $\mathrm{pH}$ and temperature stimuli have been prepared, and we demonstrate how changes to $\mathrm{pH}$ and temperature can be used to trigger the release and uptake of a hydrophobic dye.

Recent years have seen the utilization of dynamic bonds, whether they be non-covalent supramolecular interactions or so-called dynamic covalent bonds, to endow polymeric systems with the abilities to adapt their structures or compositions in response to external stimuli. ${ }^{1}$ The dynamic covalent bond ${ }^{2}$ is particularly interesting in this context, as its reversible nature enables polymeric systems incorporating these linkages to modify their architectures by reshuffling, incorporating or releasing their components, in effect providing a mechanism for polymer-based systems to reconfigure their covalent structures and therefore their functional or material properties. ${ }^{3}$ The strength of the covalent bond also ensures polymeric systems featuring this interaction possess chemical robustness.

We are interested in developing new stimuli-responsive core cross-linked star (CCS) polymers, ${ }^{4}$ a class of nano-sized polymer assembly whose structural features are defined by a core consisting of cross-linked polymer chains surrounded by a corona of polymeric arms. Much of the interest in CCS polymers is as a consequence of their architectures, possessing cross-linked cores which can be utilized as carriers for small molecules such as drugs or fragrances, and coronal chains which help to solubilize and shield the cargo from its external environment. CCS Polymers also possess solubilities and viscosities similar to linear polymers of relatively low molecular weight, additional properties which make them potentially very useful in a diverse range of fields, most notably drug delivery ${ }^{5}$ as well as imaging ${ }^{6}$ and catalysis. ${ }^{7}$

We describe here dual stimuli-responsive water-soluble CCS polymers which are prepared from cross-linking polymer chains possessing amino and aldehyde functions through the

Chemical Nanoscience Laboratory, School of Chemistry, Bedson

Building, Newcastle University, Newcastle upon Tyne, UK.

E-mail: d.a.fulton@ncl.ac.uk; Fax: + 44 (0)191222 6929;

Tel: + 44 (0) 1912227065

$\dagger$ Electronic supplementary information (ESI) available: Experimental procedures, reaction schemes, spectroscopic data, GPC traces, temperature-turbidity curves, MALLS data and DLS analysis. See DOI: $10.1039 / \mathrm{clcc} 11785 \mathrm{~h}$ formation of dynamic covalent imine bonds (Fig. 1). These CCS polymers are constructed from linear polymer chains possessing an "inert" block, which prevents macroscopic cross-linking, and a "reactive" block which contains aldehyde or amine functions which facilitate cross-linking of polymer chains through reversible imine bond formation. The reversible nature of this bond allows the position of the imine equilibrium to be modulated by changing the $\mathrm{pH}$, as imine formation is favoured at high $\mathrm{pH}$ and disfavoured at low $\mathrm{pH} .{ }^{8}$ Consequently, the assembly and disassembly of aqueous solutions of the CCS polymer can be triggered simply by changing the $\mathrm{pH}$, a process which we demonstrate can lead to the release of hydrophobic dye molecules complexed within the polymer cores. In addition to $\mathrm{pH}$ responsiveness, our CCS polymers also possess thermoresponsive cores which endows them with the ability to reversibly switch from hydrophilic to hydrophobic, and we demonstrate here the temperature controlled uptake and release of the same hydrophobic dye.

We prepared (ESI, S3 $\dagger$ ) our polymer building blocks P1 and P2b using reversible addition-fragmentation chain transfer (RAFT) polymerization. ${ }^{9}$ Polymers $\mathbf{P 1}$ and $\mathbf{P 2 b}$ were readily soluble in water, and dynamic light scattering (DLS) studies at $20{ }^{\circ} \mathrm{C}$ reveals hydrodynamic radii of $6.6 \mathrm{~nm}$ and $6.0 \mathrm{~nm}$ respectively, suggesting strongly that these polymers exist as unimolecular species in aqueous solutions and do not form micellar aggregates. This conclusion is supported by ${ }^{1} \mathrm{H}$ NMR spectroscopy (ESI, S8-9†) in $\mathrm{D}_{2} \mathrm{O}$ of each polymer, which display well-defined signals corresponding to all monomer units, signals which would be expected to diminish in intensity if the polymer chains were packed within the interior of micellar aggregates. As a consequence of their high content of $\mathrm{N}$-isopropylacrylamide monomer units, both polymers display lower critical solution temperatures (LCSTs). The LCST of P1 was measured to be $27{ }^{\circ} \mathrm{C}$, whereas the LCST of the P2b was measured to be $55{ }^{\circ} \mathrm{C}$ (ESI, S10 $\dagger$ ). The differences in measured LCST are simply on account of the relative hydrophobicity of the aromatic aldehyde functions of P1, lowering the LCST, and the hydrophilic amino functions of $\mathbf{P 2} \mathbf{b}$, which raise the LCST. Above their LCSTs, no evidence was found from DLS for the formation of micellar aggregates as the polymers precipitate from solution.

CCS Polymers were prepared by mixing equimolar solutions of $\mathbf{P 1}$ and $\mathbf{P 2 b}$ in water to afford a solution with a total polymer concentration of $1 \mathrm{wt} \%$. The $\mathrm{pH}$ was adjusted to 11.0 


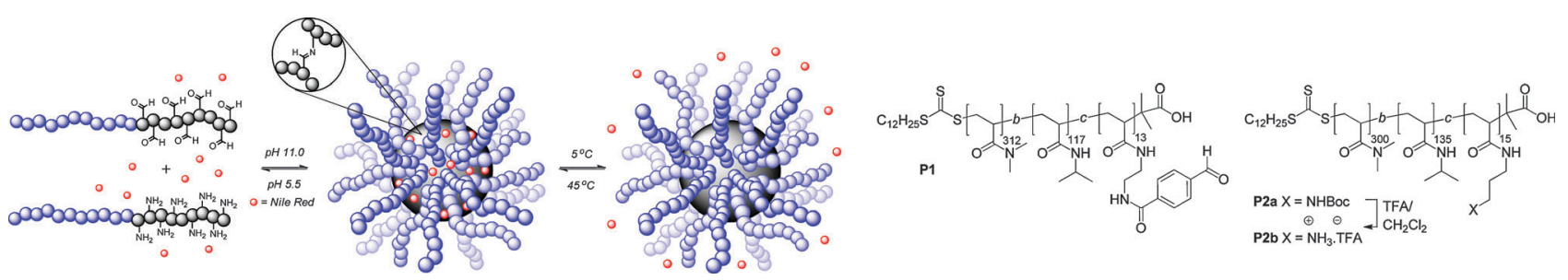

Fig. 1 Polymers possessing aldehyde and amino groups cross-link at pH 11 to form CCS polymers. Nile Red can be encapsulated within the hydrophobic core of the CCS polymer at $45^{\circ} \mathrm{C}$. The release of Nile Red can be triggered by $\mathrm{pH}$-induced disassembly of the CCS polymer system, or temperature-induced loss of the hydrophobicity of the cross-linked core. Both of these processes are reversible, and the Nile Red can be reencapsulated upon removal of the $\mathrm{pH}$ or temperature stimuli.

with small aliquots $(5 \mu \mathrm{L})$ of $1 \mathrm{M} \mathrm{NaOH}_{(\mathrm{aq})}$, and the reaction was left to equilibrate for $16 \mathrm{~h}$ at room temperature. No precipitation or macroscopic gelation was observed in the reaction mixture, an observation which suggests macroscopic cross-linking is inhibited by the 'inert' blocks within the polymer chains. Gel permeation chromatography (GPC) analysis (Fig. 2a) displayed a significant reduction in the peaks corresponding to the diblock copolymers $\mathbf{P 1}$ and $\mathbf{P} 2 \mathbf{a}^{10}$ at retention time $\sim 22.5 \mathrm{~min}$, and the appearance of a major peak indicating the formation of high molecular weight aggregates at $\sim 14 \mathrm{~min}$. The minor peaks at $\sim 20 \mathrm{~min}$ and $\sim 23 \mathrm{~min}$ are probably as a consequence of small quantities of CCS polymers possessing very low numbers of arms. ${ }^{11}$ The progress of this reaction was also followed by DLS (ESI, S1 $1 \dagger$ ) at $20^{\circ} \mathrm{C}$, which shows the disappearance of unimolecular polymeric species of $R_{\mathrm{h}}=6-7 \mathrm{~nm}$ and the formation of a larger monomodal distribution of aggregates possessing $R_{\mathrm{h}}=18.9 \mathrm{~nm}$. Multi-angle laser light scattering (MALLS) experiments (ESI, S12 $\dagger$ ) were used to estimate the $M_{\mathrm{w}}$ of the CCS polymer assembly to be $2332 \mathrm{kDa}$, suggesting that the CCS polymers possess on average about 49 arms, ${ }^{12}$ and the formation of the CCS polymer architecture was supported by determining the structure sensitive $\rho$ parameter $\left(\rho=R_{\mathrm{g}} / R_{\mathrm{h}}\right)$ defined by Burchard and co-workers ${ }^{13}$ which was found to be $\sim 1.26$, which is consistent ${ }^{14}$ with monodisperse regular star architectures. The formation of CCS polymers was also supported by ${ }^{1} \mathrm{H}$ NMR spectroscopy. The aldehyde signals of $\mathbf{P 1}$, which are evident at $\mathrm{pH}$ 5.5, disappear at $\mathrm{pH} 11.0$ suggesting that imine bond formation is occurring. Significant reductions in the intensity of the $N$-isopropylacrylamide signals relative to $N, N^{\prime}$-dimethylacrylamide were observed after the $\mathrm{pH}$ increased to 11.0 (ESI, S13†), suggesting the $N$-isopropylacrylamide units are within the interior of the CCS polymer assembly. All of these observations indicate that the polymer
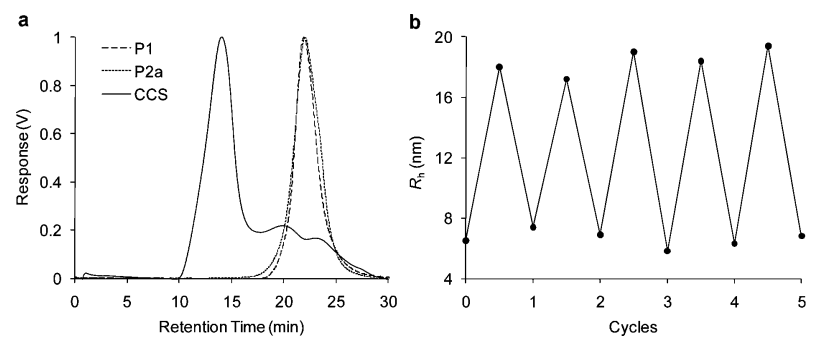

Fig. 2 (a) Differential refractive index (dRI) GPC trace (in DMF $0.6 \mathrm{~mL} \mathrm{~min}{ }^{-1}$ ) displaying the formation of CCS polymer. (b) $\mathrm{pH}-$ Triggered CCS polymer assembly and disassembly, going from $\mathrm{pH} 5.5$ (cycles $0,1,2,3,4$ and 5) to $\mathrm{pH} 11.0$ (cycles $0.5,1.5,2.5,3.5$ and 4.5). chains cross-link through imine bond formation to form CCS polymers, and are consistent with those reported previously by us for the self-assembly of CCS polymers within an analogous organic-soluble system. ${ }^{15}$

The disassembly of the CCS polymers back into their component unimolecular polymer chains was triggered by simply adjusting the $\mathrm{pH}$ of the solution to 5.5 with small aliquots $(5 \mu \mathrm{L})$ of $1 \mathrm{M} \mathrm{HCl}_{(\mathrm{aq})}$. After leaving the solution to reequilibriate for $4 \mathrm{~h}$, DLS analysis (ESI, S14 $\dagger$ ) at $20{ }^{\circ} \mathrm{C}$ showed the disappearance of the CCS polymers and the appearance of a monomodal distribution of species possessing hydrodynamic radii of 6-7 $\mathrm{nm}$ which corresponds to unimolecular polymer chains. This observation supports the idea that the CCS polymers have disassembled into their component polymer building blocks, a conclusion also supported by GPC analysis (ESI, S15†) which shows the disappearance of the large molecular weight CCS species and the reappearance of the polymer chain building blocks. The disassembly process is entirely reversible, and the ability of this polymeric system to reversibly cycle back and forth between the CCS polymer architecture and single polymer chains was demonstrated (Fig. 2b) by repeatedly switching the $\mathrm{pH}$ between 11.0 and 5.5 and monitoring the particle size distribution of the polymer solution by DLS, observations which suggest the system can be switched without any loss in switching efficiency.

To demonstrate that the CCS polymers could be kinetically fixed, the imine bonds were reduced. Excess $\mathrm{NaCNBH}_{3}$ (approx. 100 eq $\mathrm{NaCNBH}_{3}$ per imine bond) was added to a $1 \%$ wt solution of CCS polymers at $\mathrm{pH} 11.0$ and the solution was left to stir at room temperature for $2 \mathrm{~h}$. The $\mathrm{pH}$ was then adjusted to 5.5 with small aliquots $(5 \mu \mathrm{L})$ of $1 \mathrm{M} \mathrm{HCl}_{(\mathrm{aq})}$ and the sample was left overnight. DLS analysis (ESI, S16†) revealed a monomodal particle size distribution whose $R_{\mathrm{h}}=$ $19.4 \mathrm{~nm}$, suggesting that the imine bonds have been successfully reduced to amines, thus preventing the disassembly of the CCS polymer back into its constituent polymer building blocks at low $\mathrm{pH}$.

The position of the imine equilibrium is known to be sensitive to temperature, a factor which might affect the stability of these CCS polymers. To investigate this possibility, a solution of CSS polymers at $\mathrm{pH} 11.0$ were investigated at a range of temperatures $\left(5-50{ }^{\circ} \mathrm{C}\right)$ by DLS analysis (ESI, S17†), displaying near identical hydrodynamic radii across this temperature range. ${ }^{16}$ This observation confirms that any temperature-induced shift in the position of the imine equilibrium does not lead to the disassembly of CCS polymers. 

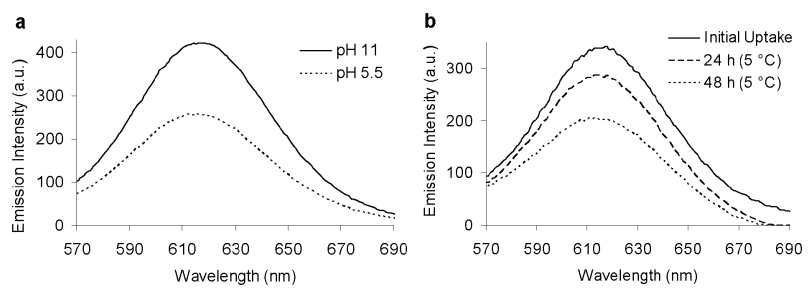

Fig. 3 (a) Fluorescence spectra displaying the $\mathrm{pH}$-triggered release of Nile Red from a solution of CCS polymers. (b) Fluorescence spectra as a function of time following the temperature-triggered release of Nile Red from a solution of CCS polymers.

We speculate that because each polymer chain is incorporated into the assembly through multiple imine linkages, then even as the equilibrium shifts away from imine formation, there are still enough imine links present to ensure the integrity of the CCS polymer assembly.

The $\mathrm{pH}$-triggered disassembly was examined further using fluorescence emission spectroscopy and the dye Nile Red as a hydrophobic probe. The CCS polymers possess cores which are composed of copolymers based upon poly- $N$-isopropylacrylamide, a thermoresponsive polymer which can undergo a reversible phase transition from hydrophilic to hydrophobic at its LCST. ${ }^{17}$ The possibility therefore exists that above their LCSTs the CCS polymers can reversibly uptake hydrophobic molecules into their cores, and then release these hydrophobes upon the $\mathrm{pH}$-triggered disassembly of the CCS polymers. Nile Red ( $1 \mathrm{mg}$ per $10 \mathrm{mg}$ of CCS polymer) was added to a $1 \mathrm{wt} \%$ solution of CCS polymer at $\mathrm{pH} 11.0$ and the resulting suspension stirred overnight at $45{ }^{\circ} \mathrm{C}$, a temperature above the estimated LCST of the CCS polymer core, followed by filtration to remove excess Nile Red. The obtained solution displayed a bright red/ purple colour (ESI, S18b $\dagger$ ), which was analyzed by fluorescence emission spectroscopy to confirm the uptake of Nile Red (Fig. 3a). The pH of the solution was then adjusted to 5.5 to trigger disassembly and the reaction was left overnight at room temperature to re-equilibrate. The solution became noticeably less intense in color (ESI, S18c $\dagger$ ) and a purple precipitate was observed. Fluorescence spectroscopy revealed a decrease in the emission intensity (Fig. 3a), observations which suggest the release of Nile Red as the CCS polymer disassembles into its unimolecular polymer chains. The reversibility of this encapsulation process was investigated by fluorescence emission spectroscopy whilst cycling the $\mathrm{pH}$ (ESI, S19†), suggesting the complexation and release of hydrophobic Nile Red dye within the core can be triggered over several cycles.

The ability to trigger in response to temperature the uptake and release of guest molecules from the thermoresponsive cores of the CCS polymers was investigated. A sample of CCS polymer containing complexed Nile Red (1 wt \% , $\mathrm{pH}$ 11.0) was stored at $5{ }^{\circ} \mathrm{C}$ for $48 \mathrm{~h}$. After this time a purple precipitate was observed and fluorescence spectroscopy (Fig. 3b) displayed a relative decrease in the emission intensity, suggesting that at low temperatures the core switches from hydrophobic to hydrophilic, releasing Nile Red from the core. The solution was then heated to $45^{\circ} \mathrm{C}$ for $24 \mathrm{~h}$ and fluorescence spectroscopy (ESI, S20 $\dagger$ ) confirmed the re-encapsulation of Nile Red. These observations suggest the cross-linked cores can reversibly switch from hydrophilic to hydrophobic, and possess the ability to complex then release hydrophobic guest molecules in response to changes in temperature.

In conclusion, we have prepared interesting water-soluble CCS polymer systems possessing $\mathrm{pH}$ - and thermoresponsiveness, and demonstrated how both of these stimuli can be used to trigger the release and uptake of a hydrophobic dye molecule. We are currently investigating the kinetics of guest uptake and release by the assembly-disassembly and hydrophilichydrophobic mechanisms, and investigating the capacities of the CCS polymers to encapsulate different guests.

Research Councils UK (fellowship to DAF), Newcastle University (Studentship to AWJ) and One North East are gratefully thanked for support.

\section{Notes and references}

1 R. J. Wojtecki, M. A. Meador and S. J. Rowan, Nat. Mater., 2011, 10, 14-27.

2 S. J. Rowan, S. J. Cantrill, G. R. L. Cousins, J. K. M. Sanders and J. F. Stoddart, Angew. Chem., Int. Ed., 2002, 41, 898-952.

3 (a) J.-M. Lehn, Prog. Polym. Sci., 2005, 30, 814-831; (b) J.-M. Lehn, Chem. Soc. Rev., 2007, 36, 151-160.

4 (a) H. Gao and K. Matyjaszewski, Prog. Polym. Sci., 2009, 34, 317-350; (b) A. Blencowe, J. F. Tan, T. K. Goh and G. G. Qiao, Polymer, 2009, 50, 5-32.

5 J. T. Wiltshire and G. G. Qiao, Aust. J. Chem., 2007, 60, 699-705.

6 K. Fukukawa, R. Rossin, A. Hagooly, E. Pressly, J. Hunt, B. Messmore, K. Wooley, M. Welch and C. J. Hawker, Biomacromolecules, 2008, 9, 1329-1339.

7 (a) T. Terashima, M. Kamigaito, K.-Y. Baek, T. Ando and M. Sawamoto, J. Am. Chem. Soc., 2003, 125, 5288-5289; (b) B. Helms, S. J. Guillaudeu, Y. Xie, M. McMurdo, C. J. Hawker and J. M. J. Fréchet, Angew. Chem., Int. Ed., 2005, 44, 6384-6387; (c) T. Terashima, M. M. Ouchi, T. Ando, M. Kamigaito and M. Sawamoto, Macromolecules, 2007, 40, 3581-3588.

8 C. Godoy-Alcántar, A. K. Yatsimirsky and J.-M. Lehn, J. Phys. Org. Chem., 2005, 18, 979-985.

9 G. Moad, E. Rizzardo and S. H. Thang, Aust. J. Chem., 2005, 59, 669-692.

10 GPC Analysis was performed on polymer 2a (the $N$-Boc derivative of $\mathbf{2 b}$ ) as polymer $\mathbf{2 b}$, like many polyacrylamides does not chromatograph well.

11 Previous work by us has identified two-armed core cross-linked species as a minor by-product of CCS polymer formation by the approach used here: see ref. 15.

12 Calculated by dividing $M_{\mathrm{w}}$ for CCS polymers by the average $M_{\mathrm{w}}$ of $\mathbf{P 1}$ and $\mathbf{P 2 b}$.

13 P. Lang, W. Burchard, M. S. Wolfe, H. J. Spinelli and L. Page, Macromolecules, 1991, 24, 1306-1314.

14 W. Burchard, M. Schmidt and W. H. Stockmayer, Macromolecules, 1980, 13, 1265-1272.

15 A. W. Jackson and D. A. Fulton, Chem. Commun., 2010, 46, 6051-6053.

16 The hydrodynamic radius of the CCS polymer would be expected to 'shrink' as the core passes through its LCST. We speculate that no 'shrinking' of the CCS polymer assembly is observed on account of the relatively high cross-linking density of the core, making the core rigid, and the relatively small size of the core, meaning any 'shrinkage' may not be within the limits of detection of dynamic light scattering.

17 H. G. Schild, Prog. Polym. Sci., 1992, 17, 163-249. 